\title{
Monitoring Coordination during Bimanual Movements: Where Is the Mastermind?
}

\author{
Julie Duque $^{\mathbf{1}}$, Marco Davare ${ }^{\mathbf{1}}$, Ludovic Delaunay ${ }^{1}$, Benvenuto Jacob ${ }^{\mathbf{1}}$, \\ Ralf Saur $^{2}$, Friedhelm Hummel ${ }^{3}$, Laurent Hermoye ${ }^{1}$, \\ Bruno Rossion $^{1,4}$, and Etienne Olivier ${ }^{1}$
}

\begin{abstract}
One remarkable aspect of the human motor repertoire is the multitude of bimanual actions it contains. Still, the neural correlates of coordinated movements, in which the two hands share a common goal, remain debated. To address this issue, we designed two bimanual circling tasks that differed only in terms of goal conceptualization: a "coordination" task that required movements of both hands to adapt to each other to reach a common goal and an "independent" task that imposed a separate goal to each hand. fMRI allowed us to pinpoint three areas located in the right hemisphere that were more strongly
\end{abstract}

\section{INTRODUCTION}

One impressive aspect of the human motor repertoire is the myriad movements it contains that require an intricate and subtle coordination between the two hands. However, so far, most bimanual studies have investigated tasks in which the movements of one hand interfere with those of the other one, leading to a "bimanual cross talk" usually observed when both hands perform actions guided by two distinct goals (Wenderoth, Puttemans, Vangheluwe, \& Swinnen, 2003; Swinnen, 2002; Serrien, Bogaerts, Suy, \& Swinnen, 1999). For example, everybody has experienced how tricky it is to draw a line with one hand and, at the same time, a circle with the other one (Franz, 1997). These studies on bimanual movements leading to cross talk have proved very useful in determining the organization, and the limits, of the neural circuit involved in such experimental conditions. However, the tasks investigated in these studies are far from being illustrative of the bimanual actions we perform daily. In fact, we are able to execute a large number of actions that require nonsymmetrical or nonsynchronous movements of both hands without experiencing any cross talk: For instance, it is very easy to cut a piece of paper while holding it with the other

${ }^{1}$ Université Catholique de Louvain, Brussels, Belgium, ${ }^{2}$ EberhardKarls University Tübingen, Germany, ${ }^{3}$ University Medical Center Hamburg-Eppendorf, Germany, ${ }^{4}$ Université Catholique de Louvain, Louvain-la-Neuve, Belgium activated in the coordination condition: the superior temporal gyrus (STG), the SMA, and the primary motor cortex (M1). We then used transcranial magnetic stimulation (TMS) to disrupt transiently the function of those three regions to determine their causal role in bimanual coordination. Right STG virtual lesions impaired bimanual coordination, whereas TMS to right M1 enhanced hand independence. TMS over SMA, left STG, or left M1 had no effect. The present study provides direct insight into the neural correlates of coordinated bimanual movements and highlights the role of right STG in such bimanual movements.

hand. The critical difference between bimanual tasks leading to a cross talk and those that do not is probably the way their goals are conceptualized (Oliveira \& Ivry, 2008; Rosenbaum, Dawson, \& Challis, 2006; Mechsner, Kerzel, Knoblich, \& Prinz, 2001). In fact, bimanual actions that do not generate cross talk rely on the integration of a unique goal (e.g., to cut a piece of paper), which guides the "coordinated" movements of both hands. In contrast, in "independent" actions, each hand has its own goal, and these two separate goals probably compete for common representational processes, leading to a substantial cross talk (Oliveira \& Ivry, 2008; Diedrichsen, Hazeltine, Kennerley, \& Ivry, 2001; Franz, Zelaznik, Swinnen, \& Walter, 2001).

So far, the specific neural correlates of coordinated bimanual movements remain unclear. Many functional imaging studies have focused on other features of bimanual movements, such as learning and complexity, or have used tasks requiring independent hand movements and were, therefore, unable to address this issue (e.g., Jantzen, Oullier, \& Scott Kelso, 2008; Puttemans, Wenderoth, \& Swinnen, 2005; Wenderoth, Debaere, Sunaert, \& Swinnen, 2005a, 2005b; Debaere, Wenderoth, Sunaert, Van Hecke, \& Swinnen, 2004b; Wenderoth, Debaere, Sunaert, van Hecke, \& Swinnen, 2004; Aboitiz, Ide, \& Olivares, 2003; De Weerd et al., 2003; Meyer-Lindenberg, Ziemann, Hajak, Cohen, \& Berman, 2002; Toyokura, Muro, Komiya, \& Obara, 1999; Sadato, Yonekura, Waki, Yamada, \& Ishii, 1997). In fact, investigating bimanual coordination per se 
is challenging because the coordination aspect has to be isolated from all other variables known to affect individual movements during bimanual tasks, such as complexity, learning stage, and feedback procedure. For this reason, the typical experimental designs that compare brain activation during bimanual movements versus their (simpler) unimanual components or late versus early learning stages are not adequate to address the issue of the neural correlates of coordination.

In the present study, we developed a new paradigm aimed at comparing two bimanual circling tasks, identical in most aspects, but that required the two hands to reach either a common (coordinated) or two separate (independent) goals. We first conducted an fMRI study to identify brain regions more active in the coordinated than in the independent condition, then we used transcranial magnetic stimulation (TMS) to induce virtual lesions of these regions to determine their causal implications in this type of bimanual coordination.

\section{METHODS}

\section{Subjects}

Fifteen healthy volunteers aged 19 to 32 years $(25 \pm$ 3.4 years) participated in the fMRI and/or TMS study. All subjects were right-handed according to the Edinburgh Handedness Inventory (score/10 = 9.6 \pm 0.7; Oldfield, 1971). Their vision was normal or corrected to normal, and none of them had a neurological history. The absence of contraindications for MRI and TMS was systematically screened. All experimental procedures were approved by the Ethics Committee of the Université Catholique de Louvain, and all subjects gave written informed consent.

\section{Bimanual Motor Tasks}

\section{Experimental Setup}

The experiment was implemented by means of the Matlab 7.0 (The Mathworks, Natick, MA) and the Cogent 2000 toolbox (FIL, LON, and ICN at the Wellcome Department of Imaging Neuroscience, London, UK). Subjects were instructed to perform continuous circular movements while holding, with each hand, a two-degree-offreedom joystick; the external joystick trajectory was limited by a circular frame. The required speed of the leftand the right-hand movements was indicated by two visual stimuli located in each half of a computer screen positioned in front of the subjects. Each visual stimulus consisted of two white balls $\left(180^{\circ}\right.$ apart) rotating around the center of each half screen (Figure 1A); we used two balls, rather than one, to incite subjects to match the speed rather than the position of the visual stimuli. During the whole trial duration, subjects had to fixate a cross displayed at the center of the screen.

Each trial lasted $24 \mathrm{sec}$ (fMRI experiment) or $12 \mathrm{sec}$ (TMS experiment) during which subjects received a con- tinuous visual feedback about the performance of each hand. This feedback consisted of a change in the background color of each half screen that was indicative of the speed of the corresponding hand with respect to the

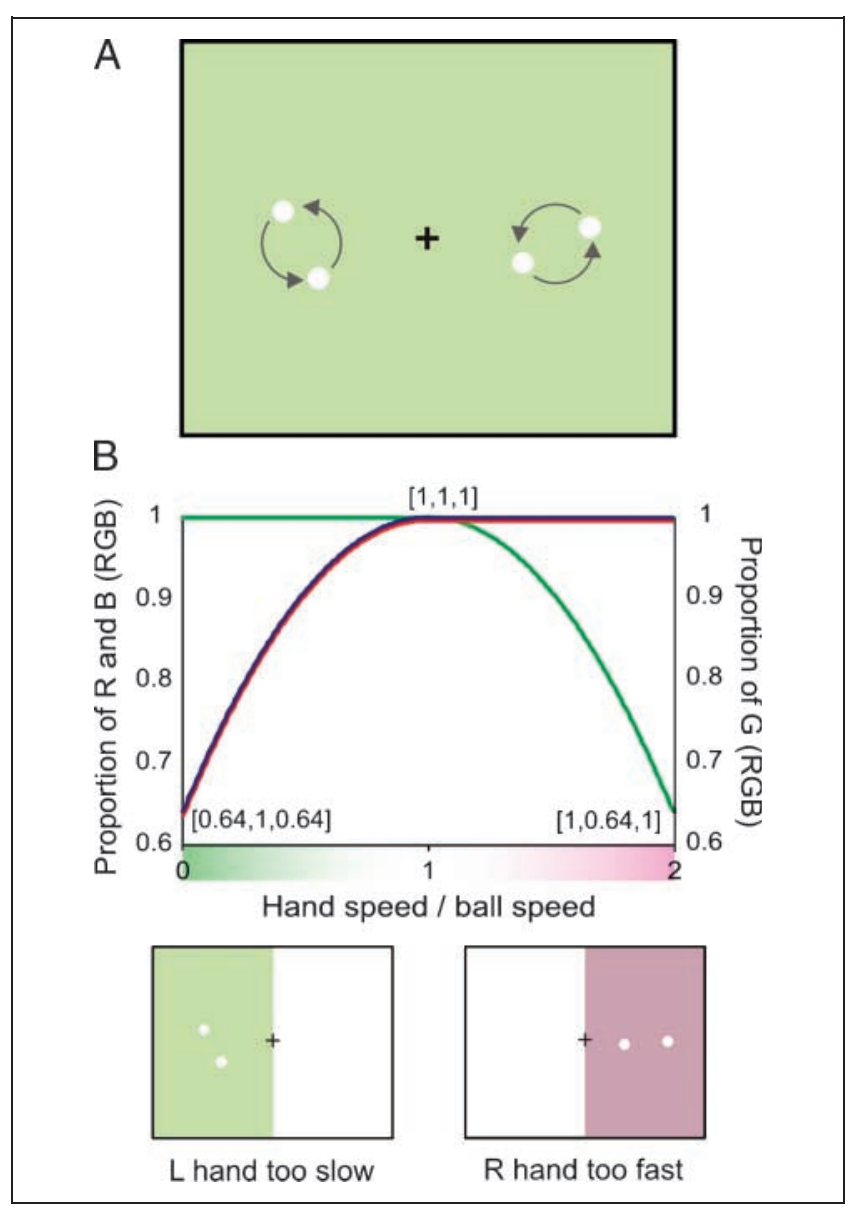

Figure 1. (A) Representation of the computer screen at the beginning of a trial. Each visual stimulus consisted of two white balls $\left(180^{\circ}\right.$ apart) moving along a circular trajectory. At the beginning of a trial, the screen background color was green because subjects were not moving yet and, therefore, too slow with respect to the corresponding rotating balls. (B) Representation of the visual feedback procedure. The background color of each half screen (left half screen for the left hand and right half screen for the right hand) varied according to the speed error of the corresponding hand circling movement. The $x$-axis represents the hand speed with respect to the target ball speed. The $y$-axis on the left represents the proportion of blue (B) and red (R) in the RGB color, whereas the $y$-axis on the right represents the proportion of green $(G)$ in the overall RGB color. The curve represents how the proportion of these three colors changed according to the hand speed error. The resulting background color is represented below the $x$-axis. Overall, a white half screen, which corresponds to the same proportion of the three colors $[1,1,1]$, indicated an adequate speed, that is, same speed as that of the corresponding balls in movement. The more the subjects were too slow, the more the half screen became green; the more the subjects were too fast, the more it became magenta. The subjects were always asked to correct their speed according to the feedback. A schematic representation of the half screen color is provided in the lower part of the figure. Note that the balls were depicted in white so that they were invisible to the subjects when the speed was correct (white background color) but reappeared as soon as it became too slow (green background color) or too fast (magenta background color). 
revolving balls (speed averaged over the last $100 \mathrm{msec}$; Figure 1B). A white background indicated that the hand speed matched perfectly that of the balls; when the speed of one hand was too low or too high, the corresponding half screen became gradually more green or magenta, respectively (see Figure 1B). The subjects were asked to take this color feedback into account and to correct the speed of their hand movements accordingly. It is noteworthy that because the balls were white, they became invisible as soon as the speed of hand movements matched that of the rotating balls. This procedure was used to force the subjects to generate circling movements internally rather than to rely continuously on the visual stimuli. Finally, during each trial, a change in the ball color was used to signal a forthcoming speed change: the balls became green or magenta to indicate, respectively, a 2-sec deceleration or a 2-sec acceleration before their speed remained constant at least for another $2 \mathrm{sec}$ (see Figure 3). These speed changes occurred twice in each trial in the fMRI experiment and only once in the TMS experiment.

\section{Coordination and Independent Tasks}

Our goal was to design two bimanual tasks that differed only in terms of goal conceptualization: a coordination task in which the movements of the two hands had to reach a common goal, that is, a given speed ratio between the two hands, and an independent task in which each hand had its own goal, that is, to move at its own speed. Because all other movement and feedback parameters were identical in both tasks, it is sensible to assume that the comparison between these two conditions should allow us to isolate the coordination factor.

Before every other trial, an imperative cue [independent (I) or coordination (C)] was displayed on the screen center for $1 \mathrm{sec}$ to indicate which task to perform for the next two consecutive trials. For the coordination task, the target speed ratio between the two hands was also displayed on the screen together with the imperative cue $\left(\mathrm{C}_{[1: 2,1: 3,2: 1 \text {, or }}\right.$ $3: 1]$ ): [1:2] and [1:3] indicated that the right hand had to turn two or three times faster than the left hand, respectively, and [2:1] and [3:1] specified the opposite ratios. Subjects were asked to comply with these speed ratios while rotating both joysticks anticlockwise. For the independent task, subjects did not receive any information about the hand speed ratio, and they had to turn the two joysticks anticlockwise independently at a speed determined by the two revolving balls displayed on each half screen.

The imperative cue, displayed for $1 \mathrm{sec}$, was followed by the rotating balls in each half screen. At the beginning of each trial, because hands were not moving yet-and were therefore always too slow-the background color was always green (see Figure $1 \mathrm{~A}$ and $\mathrm{B}$ ). Then, as mentioned above, the color of each half screen was adjusted on-line to provide a feedback about the hand speed with respect to the target speed.

As already stated, in each trial, one or two speed change (s) occurred in the TMS and the fMRI experiments, respectively. These speed changes were used to differentiate further the coordination demand in the two tasks. In the independent task, the speed of only one hand was changed at a time while keeping constant the speed of the other hand. These speed changes occurred randomly for the left or right hand, and this procedure was used to ensure that the movements of both hands remained as independent as possible. In contrast, in the coordination task, the speed changes involved the two hands simultaneously, so that the intermanual speed ratio remained constant. In this condition, subjects had imperatively to rely on the knowledge of this ratio to cope with this bimanual speed change.

Importantly, besides the difference in goal conceptualization, this study was designed to minimize the disparities between these two tasks in terms of motor and visual aspects. Indeed, despite the fact that the ball speed varied between and within trials, it was adjusted so that, on average, it was identical in both tasks (see below and Table 1). In addition, subjects were trained so that they reached a comparable level of performance in both tasks (see Results) to make sure they received the same amount of visual feedback.

Table 1. Behavioral Parameters and Subjects' Performance in the Independent and Coordination Tasks

\begin{tabular}{|c|c|c|c|c|c|c|}
\hline \multirow[b]{2}{*}{ Parameters } & \multicolumn{3}{|c|}{ Pretraining } & \multicolumn{3}{|c|}{ Posttraining } \\
\hline & Independent & Coordination & $p$ & Independent & Coordination & $p$ \\
\hline L ball speed $(\mathrm{Hz})$ & $1.3 \pm 0.1$ & $1.3 \pm 0.1$ & $n s$ & $1.3 \pm 0.1$ & $1.3 \pm 0.1$ & ns \\
\hline R ball speed (Hz) & $1.3 \pm 0.1$ & $1.3 \pm 0.1$ & ns & $1.3 \pm 0.1$ & $1.2 \pm 0.1$ & ns \\
\hline L hand speed error (\%) & $38 \pm 6.9$ & $39 \pm 7.1$ & ns & $28 \pm 4.6$ & $29 \pm 6.9$ & $n s$ \\
\hline $\mathrm{R}$ hand speed error (\%) & $33 \pm 5.9$ & $36 \pm 10.4$ & ns & $26 \pm 5.0$ & $26 \pm 6.6$ & ns \\
\hline Coordination index (\%) & $55 \pm 10$ & $56 \pm 15$ & $n s$ & $40 \pm 7.5$ & $35 \pm 5.9$ & .003 \\
\hline
\end{tabular}

$\mathrm{L}=$ left; $\mathrm{R}=$ right; hand speed error $=$ error on the speed of hand circling movements; coordination index $=$ reflects how the subjects' hand speed ratio deviates from the required (ball) speed ratio (for details, see Methods). 


\section{Signal Acquisition and Data Analysis}

The two joysticks (MACH IV; Megatron, Allinges, France) were modified to be nonferromagnetic, and the original potentiometers were replaced by two fMRI-compatible conductive plastic potentiometers. The signal from these two potentiometers allowed us to record the joystick position along the horizontal and the vertical axes. This signal was low-pass filtered to remove the electromagnetic resonance noise (Pi-section capacitive EMI/RFI low-pass filter; TUSONIX, Tucson, AZ) then amplified and filtered to prevent aliasing (Bessel fourth-order low-pass filter, cutoff frequency at $200 \mathrm{~Hz}$; Arsalis, Glabais, Belgium). These signals were then sampled at $400 \mathrm{~Hz}$ (National Instruments acquisition card, PCI-6014) on a PC for off-line analysis.

\section{fMRI Experiment}

Subjects ( $n=12,25 \pm 3.5$ years) lay down with their upper limbs next to the body and the forearms nearly vertical. In this position, they operated, with both hands, the two joysticks that were attached to an adjustable plastic desk spanning them. Visual stimuli were displayed on a computer screen projected to a mirror located in the magnet over the subject's head.

\section{Experimental Design}

Each subject was tested across three different days, which involved a familiarization session (Session 1), a training session (Session 2), and a scanning session (Session 3). In all sessions, subjects performed the tasks in a supine position to homogenize the experimental setup inside and outside the scanner. In Sessions 1 and 3, subjects performed five blocks of eight randomized trials $(4 \times$ 2 tasks). Session 2 consisted of an intensive training until subjects' performance reached a plateau, corresponding to a "hand speed error" of about 30\% (see below and Table 1). In the statistical analysis (see below), Sessions 1 and 3 were regarded as the pretraining and posttraining sessions, respectively.

\section{Scanning Procedure}

The subjects were scanned on a 1.5-T whole-body MR scanner (Philips Gyroscan Intera Scanner, Best, The Netherlands) equipped with a SENSE head coil. The functional images were acquired in five runs, each consisting of 160 gradient-echo EPI volumes that covered the whole brain (field of vision $=230 \mathrm{~mm}$, acquisition and reconstruction matrix $=64 \times 64$, slice thickness $=3.6 \mathrm{~mm}$, gap $=0 \mathrm{~mm}, 35$ axial slices, $\mathrm{TR}=3000 \mathrm{msec}, \mathrm{TE}=50 \mathrm{msec}$, flip angle $=90^{\circ}$, NSA $=1$, SENSE reduction factor $=$ 2). A high-resolution T1-weighted three-dimensional sequence was also acquired for anatomical guidance (field of vision $=230 \mathrm{~mm}$, acquisition and reconstruction matrix $=256 \times 256$, slice thickness $=1.5 \mathrm{~mm}$, gap $=0 \mathrm{~mm}$,
110 slices, $\mathrm{TR}=30 \mathrm{msec}, \mathrm{TE}=3 \mathrm{msec}$ flip angle $=30^{\circ}$, $\mathrm{NSA}=1$, SENSE reduction factor $=2$ ).

During one functional run of 160 volumes, four bimanual tasks (I, C, and two other parallel and mirror tasks not described in this article) were performed four times, each task lasting for eight volumes (24 sec). Same tasks were grouped in pairs and separated from each other by rest periods lasting four volumes $(12 \mathrm{sec})$. The occurrence of each task was randomized within each run.

\section{Behavioral Data Analysis}

Movement parameters. The speed of the left and the right hands was measured on-line by computing the first derivative of the joystick position signals. This information was used to compute the hand speed error, which provided an estimate of the ability of each hand to match the target speed, and it was calculated every $16.6 \mathrm{msec}$ $(60 \mathrm{~Hz})$ as follows:

$$
\begin{aligned}
\text { Hand speed error }= & {[\text { absolute }(\text { hand speed }} \\
& - \text { ball speed }) / \text { ball speed }] \times 100 .
\end{aligned}
$$

Another variable was defined to assess the difference between the target and the actual hand speed ratios in the two tasks. To do so, we first computed the "ball speed ratio" by expressing the speed of the fastest rotating balls with respect to that of the slowest ones, then the "hand speed ratio" was calculated by expressing the speed of the hand on the side of the fastest balls with respect to the other one. Arbitrarily, a positive ratio indicated that the right balls or the right hand was the fastest. For example, a ratio of 2 indicated that the right balls were twice faster than the left ones or that the right hand was twice faster than the left one. Then a coordination index was computed as follows:

$$
\begin{aligned}
\text { Coordination index }= & {[\text { absolute }(\text { hand speed ratio }} \\
& - \text { ball speed ratio }) / \\
& \text { ball speed ratio }] \times 100 .
\end{aligned}
$$

Because subjects were explicitly asked to control the hand speed ratio in the coordination task, we expected the coordination index to be low in that condition and higher in the independent task. This parameter should, therefore, allow us to confirm that subjects used different strategies to perform the two tasks.

For each trial, the median value of the hand speed error and the coordination index was computed both for the whole trial duration and for each phase performed at a constant speed (three per trial).

Statistical analysis. First, we performed statistics on the whole duration of trials: The hand speed error was analyzed using a three-way repeated measure ANOVA

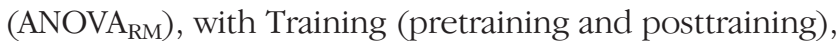
Task (independent and coordination), and Hand (left hand and right hand) as factors. Analysis of the coordination 
index was performed by using a two-way $\mathrm{ANOVA}_{\mathrm{RM}}$, with Training (pretraining and posttraining) and Task (independent and coordination) as factors. Paired $t$ tests were used for post hoc analyses.

Second, to get further insight into the task performance, we performed additional analyses on the posttraining session, that is, when subject's performance was supposed to be stable. To do so, we analyzed the movement parameters during phases of constant speed $(n=3)$ in all trials $(n=20)$ and for all subjects $(n=12)$; all these data points were pooled together $(n=720)$. The hand speed error was analyzed using a three-way ANOVA, with Task (independent and coordination), Fastest Hand (left hand and right hand), and Hand (left hand and right hand) as factors. The coordination index was analyzed by using a two-way ANOVA with factors Task (independent and coordination) and Fastest Hand (left hand and right hand). $t$ Tests were used for post hoc analyses. Values are expressed as mean $\pm S D$ throughout the manuscript.

\section{Imaging Data Analysis}

Data processing. BrainVoyager QX (Brain Innovation, Maastricht, The Netherlands) was used for fMRI data analyses. Before the statistical analysis, preprocessing consisted of linear trend removal, temporal high-pass filtering (removing frequencies lower than three cycles per run), and correction of small head movements (Friston, Frith, Turner, \& Frackowiak, 1995). The individual data were spatially and temporally smoothed using a Gaussian filter of $8 \mathrm{~mm}$ and $2.8 \mathrm{sec}$ FWHM, respectively, and transformed into Talairach space (Talairach \& Tournoux, 1988). For anatomical reference, the statistical maps computed were overlaid to the three-dimensional T1-weighted scans.

Statistical analysis. In each subject, predictors for the two experimental conditions [independent (I) and coordination (C)] were obtained by convolution of an ideal boxcar response with a linear model of the hemodynamic response (Boynton, Engel, Glover, \& Heeger, 1996). For group analyses, a random effect general linear model was used, and statistical maps were derived from the resulting $t$ values associated with each voxels. First, the global network involved in the execution of the bimanual tasks was determined by identifying all areas that showed significant activation during the movements in $\mathrm{C}$ and I with respect to rest $(\mathrm{R})$, that is, $(\mathrm{I}-\mathrm{R})$ and $(\mathrm{C}-\mathrm{R})$. The obtained $p$ values were corrected for multiple comparisons following the false discovery rate procedure (Genovese, Lazar, \& Nichols, 2002) with a probability of false detection set at $q=0.02$, which corresponded to a $p<.001$. The minimum cluster size was set at 50 voxels.

In addition, conjunction analyses (Price \& Friston, 1997) were used to identify those voxels showing (1) a higher activity in $\mathrm{C}$ than $\mathrm{I}$ and being positively activated in $\mathrm{C}$ with respect to $\mathrm{R}[(\mathrm{C}-\mathrm{R}) \cap(\mathrm{C}-\mathrm{I})]$ and (2) a higher activity in I than $\mathrm{C}$ and being positively activated in I with respect to $R[(I-R) \cap(I-C)]$. Statistical parametric maps were derived from the resulting $t$ values associated with each voxel and thresholded at $p<.001$ (uncorrected for multiple comparisons) with a cluster size $>50$. We choose this uncorrected threshold because both the random effect analysis and the method for identifying functional regions by specifying conjunctions (Friston, Penny, \& Glaser, 2005; Nichols, Brett, Andersson, Wager, \& Poline, 2005) are highly conservative techniques. The maximum $t$ values for each cluster are reported in the relevant tables.

\section{TMS Experiment}

The TMS experiment was performed about 5 months after the fMRI study. The subjects ( $n=9,24 \pm 3.1$ years), six of whom had already participated in the fMRI experiment, sat $50 \mathrm{~cm}$ in front of a computer screen with their upper limbs next to the body and the hands holding two joysticks attached to a tilted plastic desk as in the fMRI experiment; the elbows were flexed and the forearms were supported by a pillow. As in the fMRI experiment, the subjects underwent a training session before the TMS experiment to make sure they all reached the same level of performance. This procedure was of course necessary for the three new subjects, although they were members of the laboratory and had already practiced this task before, and also for the six subjects having participated in the fMRI experiment because of the long delay between the two experiments. Each trial lasted for $12 \mathrm{sec}$ during which subjects were asked to perform the same bimanual motor tasks as in the fMRI experiment. However, as already mentioned, in the TMS experiment, only one speed change occurred per trial. TMS was always time locked to the onset of the speed change to make sure that the interference induced by TMS occurred when the coordination or the independence demand was the highest.

\section{Experimental Design}

We investigated the effect of virtual lesions induced by repetitive TMS (rTMS; $10 \mathrm{~Hz}, 500 \mathrm{msec}$ ) applied over the brain regions in which the fMRI showed a stronger activation in the coordinated than in the independent task, namely, right SMA, right primary motor cortex (M1), and right superior temporal gyrus (STG). The left M1 and the left STG were chosen as control sites for unspecific TMS effects.

The experiment was divided into 15 blocks of 30 trials (3 blocks for each stimulation site); each block lasted about 6 min. During each block, subjects performed 12 coordinated and 18 independent trials; in half of the trials, rTMS was delivered at the onset of the speed change. Each experiment consisted of two sessions performed on separate days: In the first one, the left and the right M1 were stimulated (6 blocks), and in the second one, rTMS was applied over the right SMA, the right STG, and the left STG (9 blocks). All rTMS sites were counterbalanced within subjects. The main experiment was always preceded by a training session lasting at least half an hour. 


\section{Transcranial Magnetic Stimulation}

TMS was delivered with a rapid model 200 stimulator (Magstim, Whitland, UK) through a 70-mm figure-ofeight coil. To target the SMA, the coil was held tangential to the skull with the handle pointing backward (MeyerLindenberg et al., 2002), and for M1 and STG, the coil was positioned with the handle pointing backward and laterally at a $45^{\circ}$ angle away from the midline. Before each experiment, TMS was applied over both M1, and the coil position was adjusted to optimize, in the contralateral first dorsal interosseus (1DI), the motor-evoked potential (MEP) amplitude in response to a single TMS pulse. Once the optimal coil position was found, we determined the resting motor threshold (rMT), defined as the minimum intensity that induced $50 \mu \mathrm{V}$ peak-to-peak MEPs in 5 of 10 trials (Rossini et al., 1994). The rMT was measured separately for the right and the left 1DI and was averaged. Importantly, there was no significant difference between the rMT in the two M1 ( $48 \pm 6.8 \%$ and $48 \pm 7.1 \%$ of stimulator output for the left and the right $\mathrm{M} 1$, respectively). The intensity of stimulation was set at $120 \%$ of this mean value for both STG and SMA but only at 80\% for M1 to avoid disturbing movement execution. This later point was confirmed by questioning the subject after the first block of M1 stimulation. rTMS trains were separated by at least 8 sec (Wassermann, 1998).

\section{Location of Stimulation Sites}

As mentioned above, for M1 stimulation, the coil was located at the optimal position to elicit MEPs in the contralateral 1DI. The location of the coil over right SMA and right STG was determined by using the mean Talairach coordinates of the activation peaks obtained from the coordination-independent contrast. These coordinates were then transformed ("denormalization") to fit the native anatomical MRI of each individual. The coil was precisely positioned by means of an original method that allowed us to perform an on-line coregistration of the stimulation sites onto individual anatomical MRI (Davare, Andres, Clerget, Thonnard, \& Olivier, 2007; Davare, Andres, Cosnard, Thonnard, \& Olivier, 2006; Noirhomme et al., $2004)$. The actual Talairach coordinates $(x, y, z)$ of stimulation points were $1.4 \pm 2.4,-10.2 \pm 8.6$, and $67.5 \pm 4.9$ (mean $\pm S D ; n=9$ ) for the SMA and 67.9 $\pm 2.1,-35.2 \pm$ 7.4 , and $16.0 \pm 7.2$ and $-64.4 \pm 3.6,-41.7 \pm 6.6$, and $18.7 \pm 8.7$ for the right and the left STG, respectively (see Figure 2).

\section{Behavioral Data Analysis}

Movement parameters. The ability of the subjects to cope with speed changes of rotating balls was estimated by fitting a regression line on the velocity trace of each hand during these transitory phases lasting $2 \mathrm{sec}$. We then computed a "hand slope error" by comparing the slope of the regression lines calculated for the hands with that calculated for the corresponding rotating balls:

Hand slope error $=[$ absolute $($ hand slope - ball slope $)]$

In addition, we measured the difference between the slopes computed for the two hands ("hand slope difference") as well as the difference between the slopes computed for the two corresponding balls ("ball slope difference"). To do so, we subtracted the slope computed for the fastest ball from that calculated for the slowest one, and we performed the same measurement for the

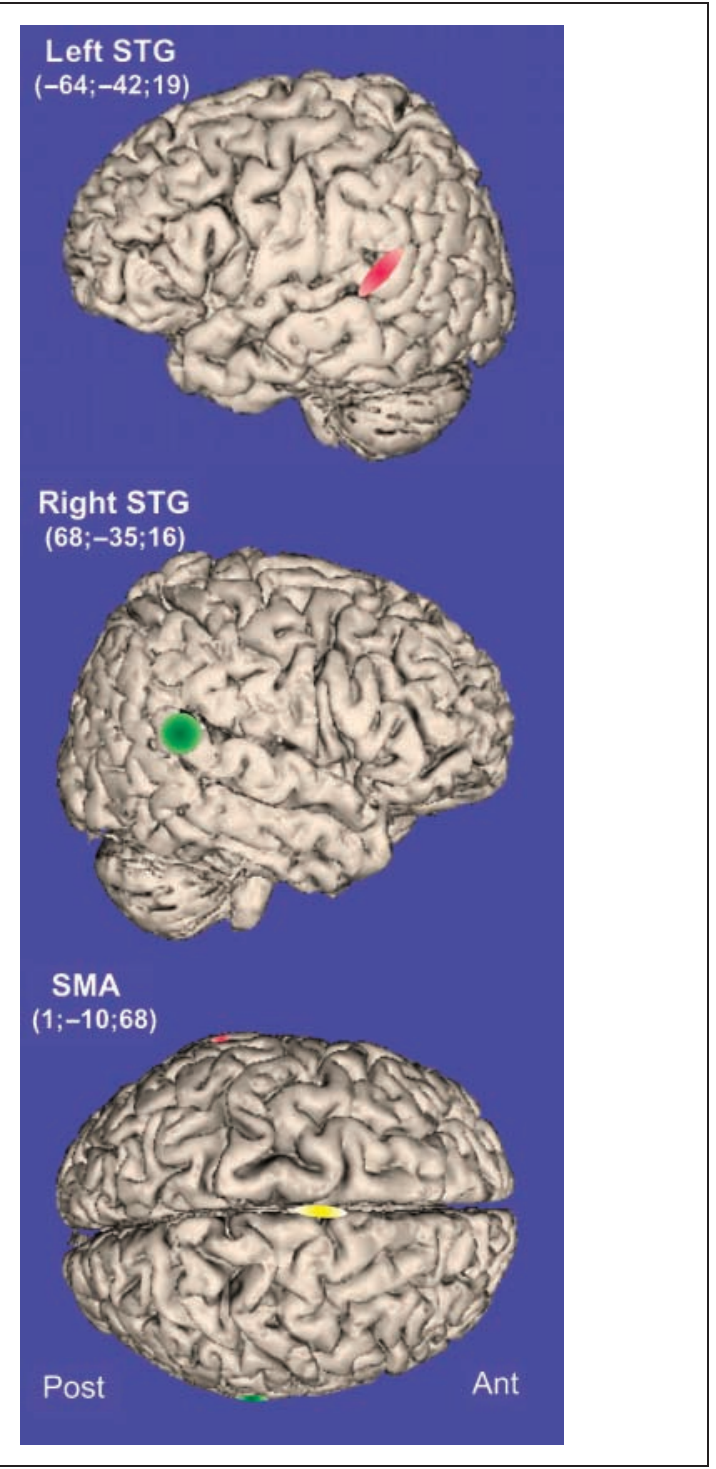

Figure 2. Location of the TMS coil positions used to induce virtual lesions of left (red) and right (green) STG and of SMA (yellow). Each ellipse is centered on the mean Talairach coordinates of the stimulation points, and their surface shows the $95 \%$ confidence interval of the normalized coordinates calculated for each subject. The loci of left and right M1 stimulations were determined according to the amplitude of MEPs in a hand muscle and were, therefore, not coregistered on the subjects' brain. 
corresponding hand slopes, for example, if the left ball was the fastest, ball slope difference $=[$ absolute (left ball slope - right ball slope)] and hand slope difference $=$ [absolute (left hand slope-right hand slope)]. This allowed us to compute a "slope coordination index" defined as follows:

$$
\begin{aligned}
\text { Slope coordination index }= & {[\text { absolute }(\text { hand slope diff }} \\
& - \text { ball slope diff })] .
\end{aligned}
$$

This parameter informed us about the subject ability to maintain a correct hand speed relationship during the speed changes. As for the coordination index in the fMRI experiment, we expected this index to be smaller in the coordination than independent task.

Statistical analysis. First, in control trials (no TMS), the hand slope error was analyzed using a two-way ANOVA $_{R M}$, with Task (independent and coordination) and Hand (left and right) as factors, and the slope coordination index was analyzed using a one-way ANOVA $\mathrm{RM}_{\mathrm{M}}$, with the Task (independent and coordination) as a factor. Then, for each TMS site, the hand slope error was analyzed using a three-way $\mathrm{ANOVA}_{\mathrm{RM}}$, with Task (independent and coordination), TMS (TMS and no TMS), and Hand (left and right) as factors, and the slope coordination index was analyzed using two-way ANOVAs $s_{\mathrm{RM}}$, with Task (independent and coordination) and TMS (TMS and no TMS) as factors. Paired $t$ tests were used for post hoc analyses. Values are expressed as mean $\pm S D$.

\section{RESULTS \\ fMRI Experiment}

\section{Behavioral Results}

During the pretraining session, all subjects $(n=12)$ had major difficulties in performing the two tasks: They showed a strong tendency to rotate the two joysticks at the same speed and could barely dissociate the movements of the two hands (Figure 3, left). Furthermore, in the coordination task, subjects were unable to maintain the target speed ratios between the two hands. Then, progressively in the course of the training session, subjects succeeded in integrating the target speed ratios in the coordination task (Figure 3, right) and in overcoming the bimanual cross talk in the independent task.

Consistently, the ANOVA ${ }_{\mathrm{RM}}$ showed a significant main effect of Training on both the hand speed error $(F=34.0$, $p<.001)$ and the coordination index $(F=47.8, p<.001$; Table 1). Importantly, we found that the hand speed error was identical in both tasks (main effect of Task: $F=0.7$, $p=.4$; see Table 1 ), showing that both tasks were executed with the same accuracy when considering the performance of each hand individually. It is noteworthy that, in the two tasks, the hand speed error was always larger for the left than for the right hand (main effect of Hand:

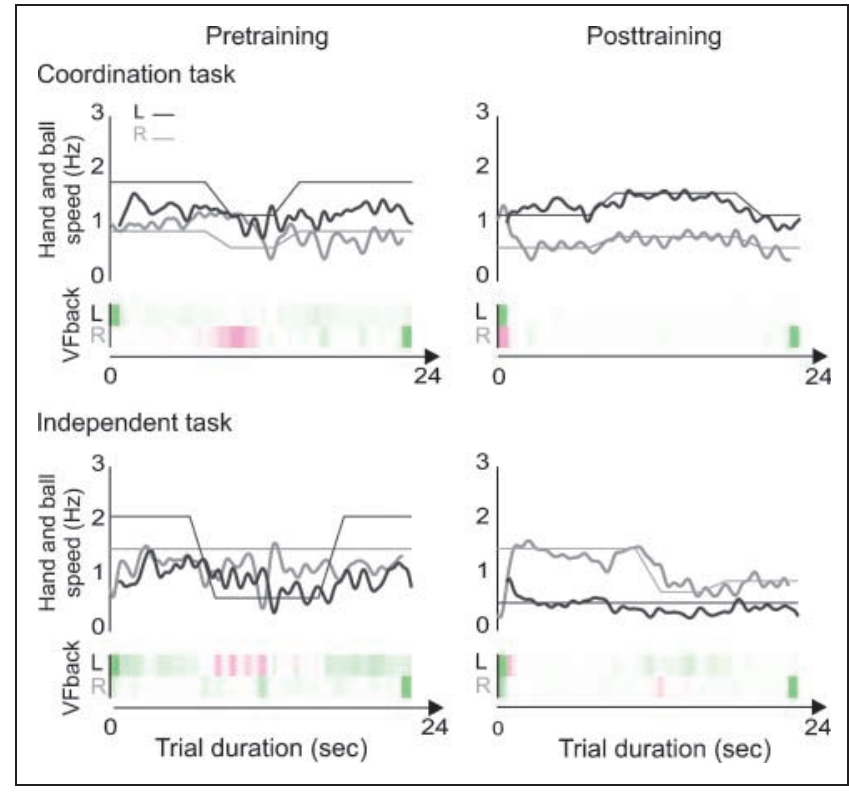

Figure 3. Representative trials showing the performance of one subject in the coordination (upper trace) and independent (lower trace) tasks during the pretraining (left) and posttraining (right) sessions. The ball speed (thin straight lines) and the subject speed (larger lines) are depicted in gray for the right (R) hand and in black for the left (L) hand. Note the difficulty that the subject showed in dissociating both hand movements when he first performed the tasks (left) but then the correct matching of the ball speeds following the training (right) for both tasks. The visual feedback (VFback) given to the subject throughout the trials is shown below the corresponding conditions. Note the whiter background color of the $\mathrm{L}$ and $\mathrm{R}$ half screens after the training in the two tasks.

$F=16.1, p=.002$; see Table 1$)$. In contrast, as far as the coordination index is concerned, we found a significant Training $\times$ Task interaction $(F=5.4, p=.04$; Table 1$)$. In the pretraining session, the coordination index was large and identical in both tasks (paired $t=0.2, p=.8$ ). However, the coordination index was influenced differentially by training and became smaller in the coordination than in the independent task (paired $t=3.8, p=.003$ ). This indicates that, although the individual performance of each hand was identical in both tasks, the coupling between the two hands was greater in the coordination task. This finding allows us to assume that subjects used different strategies to perform the two tasks investigated in the present study.

To compare further the two tasks, we analyzed the hand speed ratio and the coordination index during the three phases of constant speed of trials performed after training, that is, during the scanning session (see Methods). Individual data points for the hand speed ratio and the coordination index, sorted as a function of the ball speed ratio, are shown in Figure 4A and B, respectively. The hand speed ratio matched better the ball speed ratio in the COordination than in the independent task, as indicated by a smaller coordination index in the coordination (16\%) than in the independent task (33\%; see Figure 4B; ANOVA, 
Figure 4. Individual data for the hand speed ratio (A) and coordination index (B) as well as the mean and $S D$ of hand speed error $(C)$ for all the phases of constant speed $(n=$ 60) of all subjects $(n=12)$, as a factor of the ball speed ratio (A), for the coordination (left) and the independent (right) tasks. A ratio $<0$ represents conditions where the left hand was faster than the right hand whereas a ratio $>0$ represents the reverse conditions. Abs = absolute; $* p<.05 ; \mathrm{L}=$ left $; \mathrm{R}=$ right

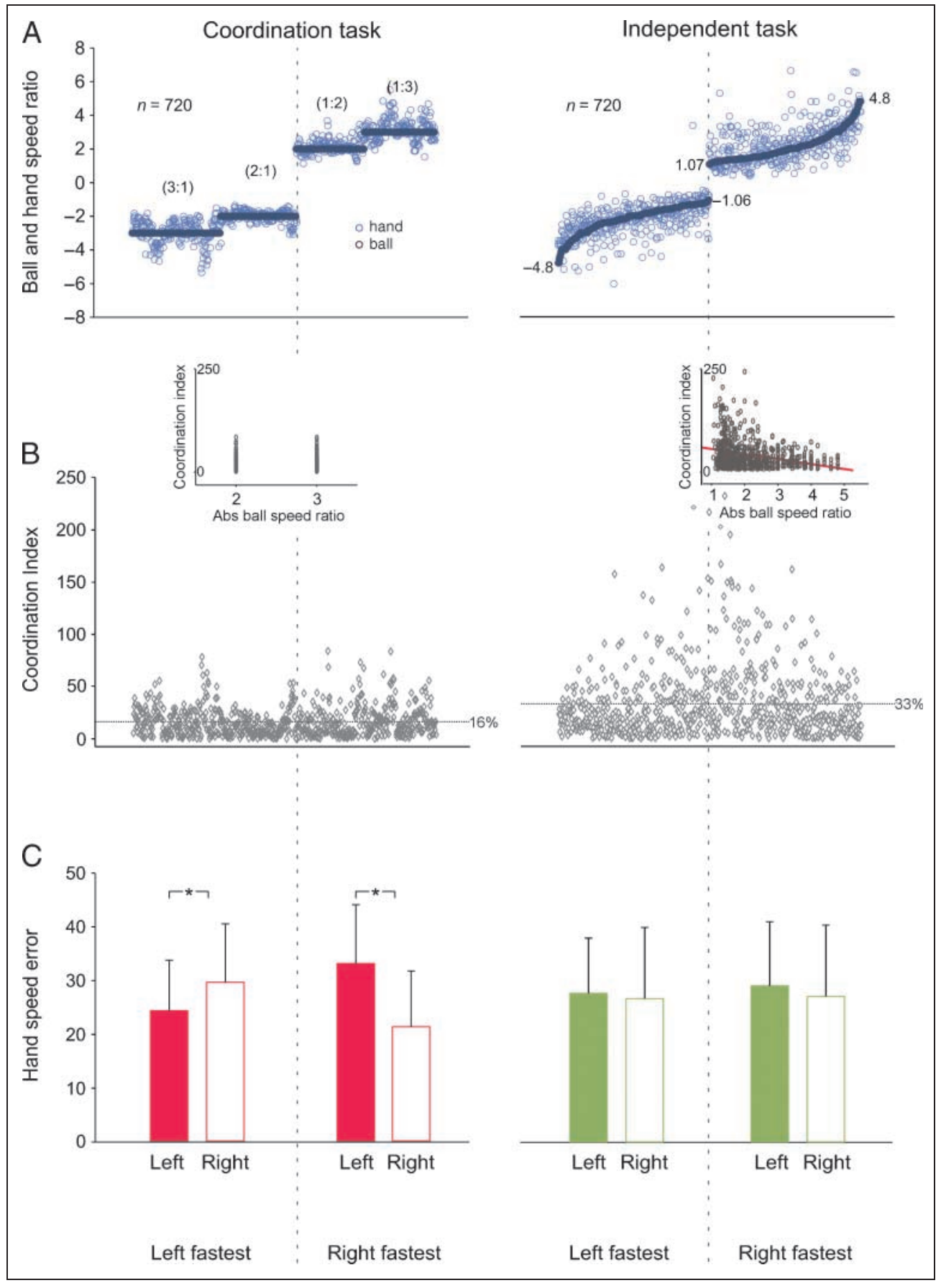

$F=130.68, p<.001)$. In the coordination task, the coordination index was not different for the two target ratios (see inset; Figure 4B). In the independent task, the coordination index increased when ratios got closer to 1 , probably because the smaller difference between the speed of both hands made it more difficult to overcome the tendency to perform synchronous movements. This result might highlight the importance of explicit cues to guide separate movements of the two hands during bimanual independent tasks. Importantly, in the independent task, the coordination index was not higher for noninteger ball speed ratios (e.g., 2.5, 3.5, 4.5) than for integer ratios (e.g., 2, 3, 4; see inset of Figure 4B). This finding rules out the possibility that the higher coordination index found in the independent task was due to the presence of more complex, noninteger ratios when compared with the coordination task.

Further evidence that subjects used distinct strategies to perform the two tasks comes from the differential influence of the relative hand speed on the hand speed error (Figure 4C). Indeed, the ANOVA showed a significant Task $\times$ Fastest Hand $\times$ Hand interaction $(F=89.47, p<.001)$ on the hand speed error: Whereas the hand speed error was systematically smaller for the fastest hand in the coordination task ( $t$ tests, all $p<.001$ ), no such effect was found in the independent task (see Figure 4C). 


\section{Imaging Results}

Brain activation during bimanual tasks versus rest. We first identified the entire network activated during bimanual movements. To do so, we determined the regions showing a stronger activation during the bimanual tasks than in the rest condition (see Table 2). We found a bilateral network, including the primary sensorimotor hand area (S1/M1) extending to the dorsal premotor cortex, SMA, inferior parietal gyrus, basal ganglia, and cerebellum. In addition, in the right hemisphere, we found an activation of the ventral premotor cortex, the dorsolateral prefrontal cortex, and the middle and superior temporal gyri (MTG and STG; see Table 2). This bimanual network is consistent with that reported in previous studies (for a review, see Swinnen \& Wenderoth, 2004).

Areas specifically involved in the coordination task. We found three areas in the right hemisphere that showed a greater activation in the coordination than in the independent task, namely, M1, $t(11)=5.23, p=.0003$, SMA, $t(11)=5.14, p=.0003$, and STG, $t(11)=5.85, p=$ .0001 (see Table 3 and Figure 5).

Areas specifically involved in the independent task. Areas showing a stronger activation in the independent than in the coordination task were the right inferior parietal gyrus, $t(11)=5.80, p=.0001$, MTG, $t(11)=6.69, p=$ .00003 , and right fusiform gyrus, $t(11)=5.84, p=.0001$ (see Table 3 and Figure 6).

\section{TMS Experiment}

In control trials (no TMS), we found, consistent with fMRI data, that the hand speed error computed during the phases of constant speed was comparable in both tasks: The hand speed error was $16.4 \pm 5.8 \%$ and $18.5 \pm 13.9 \%$ for the left and the right hands, respectively, in the coordination task and $16.4 \pm 3.7 \%$ and $16.2 \pm 3.4 \%$ for the independent task $(n=9)$. In contrast, we found that the hand slope error was larger in the independent than in the coordinated task, suggesting that speed changes were easy to perform when occurring in both hands simultaneously (ANOVA ${ }_{\mathrm{RM}}$, main Task effect, $F=29.6, p=$ .001). Consistently, the slope coordination index (see Methods) was also higher in the independent than in the coordination task $(F=44.5, p<.001)$.

Virtual lesions of the right STG specifically modified the hand slope error in the coordination task, without affecting any movement parameters in the independent task. ANOVA $_{\text {RM }}$ showed a significant effect of Task $(F=11.3$, $p=.01)$, TMS $(F=9.8, p=.02)$, Hand $(F=16.0, p=$ $.004)$, and a significant Task $\times$ TMS interaction $(F=5.6$, $p=.04$ ) on the hand slope error (see Figure 7A). Post hoc analyses indicated that the virtual lesions of the right STG increased the hand slope error in the coordination task with respect to the no TMS condition, for both the left hand (paired $t$ test, $p=.057)$ and the right hand $(p=.001)$.
TMS applied over the right $\mathrm{M} 1$ yielded a significant effect of Task (ANOVA $\left.{ }_{\mathrm{RM}}, F=23.5, p=.001\right)$, TMS $(F=7.6$, $p=.025)$, Hand $(F=11.7, p=.009)$, and a Task $\times$ TMS interaction $(F=10.6, p=.01)$ on the hand slope error. In particular, we found a significant decrease in the hand slope error of the left hand following right M1 virtual lesions but only in the independent task (paired $t$ test, $p=.01 ;$ Figure $7 \mathrm{~B}$ ). In other words, a transient disruption of the right M1 function improved the ability of the left hand to adapt to the speed changes in the independent task but had no effect on the coordination task performance. ANOVA $A_{\mathrm{RM}}$ also showed a significant Task $\times$ TMS interaction $(F=8.1, p=.022)$ on the slope coordination index, and post hoc analyses indicated that rTMS applied over right M1 decreased the slope coordination index during the speed change only in the independent task $(p=$ .006; Figure 7B).

No effects were found after virtual lesions of SMA or when rTMS was applied over the control sites (left M1 and left STG).

\section{DISCUSSION}

The aim of the present study was to determine the neural correlates of one of the most critical—and probably the least understood-processes underlying skilled actions, that is, the coordination between both hands during the performance of bimanual movements. Our fMRI results show that three cortical areas, all located in the right hemisphere, are more active in a coordination task than in an independent task, namely, the STG, the SMA, and the M1. The causal relevance of the activity in these three regions for the bimanual coordination was then investigated by using TMS to interfere transiently and reversibly with their function.

\section{Neural Correlates of Bimanual Coordination}

Many functional imaging studies have already examined the neural correlates of bimanual movements (Puttemans et al., 2005; Debaere et al., 2001, 2004b; De Weerd et al., 2003; Debaere, Wenderoth, Sunaert, Van Hecke, \& Swinnen, 2003; Meyer-Lindenberg et al., 2002; Toyokura et al., 1999; Sadato et al., 1997) with a particular interest for independent tasks (Wenderoth et al., 2004, 2005a, 2005b). However, to our knowledge, very few studies have tried to identify the cortical areas dealing specifically with the coordination aspect of bimanual movements (Jantzen et al., 2008; Debaere et al., 2003).

Our finding that right STG was more activated in the coordination than in the independent task was unexpected because its contribution to bimanual movements has never been really emphasized before, although many studies reported an activation of this area in complex spatial or rhythmic coordination movements (Oullier, Jantzen, Steinberg, \& Kelso, 2005; Debaere et al., 2004b; Ullen, Forssberg, \& 
Table 2. General Motor Network Activated during the Bimanual Tasks

\begin{tabular}{|c|c|c|c|c|c|c|c|c|}
\hline \multirow[b]{3}{*}{ Brain Regions } & \multicolumn{4}{|c|}{ Coordination Task } & \multicolumn{4}{|c|}{ Independent Task } \\
\hline & \multicolumn{3}{|c|}{$\begin{array}{c}\text { Peak Activation } \\
\text { Coordinates }\end{array}$} & \multirow[b]{2}{*}{$t(11)$} & \multicolumn{3}{|c|}{$\begin{array}{c}\text { Peak Activation } \\
\text { Coordinates }\end{array}$} & \multirow[b]{2}{*}{$t(11)$} \\
\hline & $x$ & $y$ & $z$ & & $x$ & $y$ & $z$ & \\
\hline \multicolumn{9}{|l|}{ Frontal lobe } \\
\hline \multirow[t]{2}{*}{ R sensorimotor region (BA 3/4), S1/M1 } & 27 & -31 & 46 & 8.95 & 33 & -25 & 49 & 9.00 \\
\hline & & & & & 48 & -19 & 37 & 11.13 \\
\hline \multirow[t]{2}{*}{$\mathrm{L}$} & -33 & -22 & 46 & 8.60 & -33 & -22 & 46 & 8.68 \\
\hline & & & & & -48 & -19 & 46 & 7.37 \\
\hline $\mathrm{R}$ inferior frontal gyrus (BA 44), PMv & 42 & 17 & 22 & 10.19 & 45 & 11 & 22 & 9.71 \\
\hline (BA 45), DLPFC & 36 & 35 & 13 & 6.67 & 39 & 32 & 10 & 6.89 \\
\hline R middle frontal gyrus (BA 46) & 34 & 28 & 25 & 10.00 & 30 & 29 & 25 & 11.46 \\
\hline R superior frontal gyrus (BA 6), SMA & 3 & -25 & 46 & 5.70 & 1 & -25 & 46 & 4.96 \\
\hline \multirow[t]{2}{*}{ L/MID } & 0 & -25 & 49 & 5.34 & 0 & -22 & 49 & 5.01 \\
\hline & & & & & -3 & -13 & 58 & 4.85 \\
\hline \multirow[t]{2}{*}{ L superior frontal gyrus (BA 4/6), M1 and PMd } & -30 & -16 & 64 & 6.22 & -27 & -16 & 61 & 7.41 \\
\hline & -18 & -25 & 61 & 5.67 & -18 & -25 & 61 & 6.07 \\
\hline \multicolumn{9}{|l|}{ Parietal lobe } \\
\hline \multirow[t]{3}{*}{ R inferior parietal gyrus (BA 39/40) } & 30 & -58 & 37 & 7.77 & 33 & -55 & 34 & 8.29 \\
\hline & 42 & -46 & 46 & 10.93 & 45 & -40 & 46 & 9.10 \\
\hline & 54 & -34 & 25 & 6.77 & & & & \\
\hline \multirow[t]{2}{*}{$\mathrm{L}$} & -45 & -43 & 40 & 7.50 & -45 & -43 & 40 & 9.46 \\
\hline & -24 & -64 & 31 & 4.92 & -27 & -58 & 46 & 5.94 \\
\hline \multicolumn{9}{|l|}{ Temporal lobe } \\
\hline R MTG (BA 37) & 45 & -64 & 4 & 9.14 & 45 & -64 & 4 & 10.84 \\
\hline R STG (BA 22) & 39 & -46 & 4 & 6.88 & 36 & -40 & 7 & 7.28 \\
\hline \multicolumn{9}{|l|}{ Occipital lobe } \\
\hline \multirow[t]{2}{*}{ R middle occipital gyrus (BA 18/19) } & & & & & 39 & -64 & -5 & 9.46 \\
\hline & & & & & 27 & -79 & 22 & 4.94 \\
\hline \multirow[t]{2}{*}{$\mathrm{L}$} & -27 & -67 & -14 & 7.35 & -27 & -79 & 4 & 6.14 \\
\hline & -15 & -82 & -2 & 5.62 & -24 & -76 & -8 & 8.14 \\
\hline \multicolumn{9}{|l|}{ Basal ganglia } \\
\hline \multirow[t]{2}{*}{$\mathrm{R}$} & & & & & 9 & 8 & 1 & 5.90 \\
\hline & & & & & 18 & -4 & 16 & 4.63 \\
\hline \multirow[t]{2}{*}{$\mathrm{L}$} & -21 & 8 & 25 & 6.68 & -18 & 5 & 25 & 6.55 \\
\hline & -15 & 2 & 4 & 6.09 & & & & \\
\hline
\end{tabular}


Table 2. (continued)

\begin{tabular}{|c|c|c|c|c|c|c|c|c|}
\hline \multirow[b]{3}{*}{ Brain Regions } & \multicolumn{4}{|c|}{ Coordination Task } & \multicolumn{4}{|c|}{ Independent Task } \\
\hline & \multicolumn{3}{|c|}{$\begin{array}{c}\text { Peak Activation } \\
\text { Coordinates }\end{array}$} & \multirow[b]{2}{*}{$t(11)$} & \multicolumn{3}{|c|}{$\begin{array}{c}\text { Peak Activation } \\
\text { Coordinates }\end{array}$} & \multirow[b]{2}{*}{$t(11)$} \\
\hline & $x$ & $y$ & $z$ & & $x$ & $y$ & $z$ & \\
\hline \multicolumn{9}{|l|}{ Cerebellum } \\
\hline \multirow[t]{2}{*}{$\mathrm{R}$ cerebellar hemisphere } & 21 & -58 & -17 & 11.05 & 21 & -58 & -20 & 14.10 \\
\hline & & & & & 30 & -61 & -35 & 5.81 \\
\hline $\mathrm{L}$ & -15 & -46 & -17 & 14.82 & & & & \\
\hline \multirow[t]{2}{*}{$\mathrm{R}$ vermis } & 6 & -52 & -17 & 16.78 & 6 & -52 & -17 & 15.86 \\
\hline & 1 & -61 & -2 & 6.03 & 1 & -61 & -2 & 7.12 \\
\hline $\mathrm{L}$ & 0 & -52 & -17 & 15.54 & 0 & -55 & -17 & 14.19 \\
\hline
\end{tabular}

Location of peak activations and $t$ values for the coordination and independent tasks (false discovery rate $<0.02$ ); $\mathrm{L}=$ left; $\mathrm{R}=$ right; $\mathrm{M} 1=$ primary motor cortex; PMv = ventral premotor cortex; PMd = dorsal premotor cortex; DLPFC = dorsolateral prefrontal cortex; SI = somatosensory cortex; $\mathrm{MTG}=$ middle temporal gyrus; STG = superior temporal gyrus; $\mathrm{MD}=$ middle.

Ehrsson, 2003; Mayville, Jantzen, Fuchs, Steinberg, \& Kelso, 2002). In contrast, the possible contribution of right STG, especially its posterior part, to spatial attention has been much debated (Gharabaghi, Fruhmann Berger, Tatagiba, \& Karnath, 2006; Meister et al., 2006; Karnath et al., 2005; Thiebaut de Schotten et al., 2005; Ellison, Schindler, Pattison, \& Milner, 2004; Mort et al., 2003; Karnath, 2001; Karnath, Ferber, \& Himmelbach, 2001). In the present study, it may be argued that the coordination task required a continuous

Table 3. Localization of Peak Activations (Talairach Coordinates) and $t$ Values for the Areas Showing a Different Activation Level for the Coordination and the Independent Tasks

\begin{tabular}{lrrrr}
\hline & \multicolumn{3}{c}{$\begin{array}{c}\text { Peak Activation } \\
\text { Coordinates }\end{array}$} \\
\cline { 2 - 4 } Brain Regions & $x$ & $y$ & $z$ & $t(11)$ \\
\hline$C>I$ & 31 & -27 & 50 & 5.23 \\
R primary motor cortex (BA 4) & 4 & -25 & 48 & $5.14^{*}$ \\
R SMA (BA 6) & 36 & -45 & 10 & 5.85 \\
R STG (BA 22) & & & & \\
& & & & \\
$I>C$ & 48 & -65 & 2 & 6.69 \\
R MTG (BA 37) & 30 & -51 & 41 & 5.80 \\
R inferior parietal gyrus (BA 40) & 29 & -48 & -13 & 5.84 \\
R fusiform gyrus (BA 19) & & & & \\
\hline
\end{tabular}

$\mathrm{C}=$ coordination task $\mathrm{I}=$ independent task; $t$ values indicate the comparison for the contrast with the largest signal difference $(p<.001$, uncorrected).

*Threshold defined at $p<.002$ (uncorrected) for a cluster size $>50$ voxels monitoring of the spatial location of the two hands to keep them synchronized to maintain the required hand speed ratio. In fact, the contribution of right STG to this mechanism is compatible with its potential role in spatial attention and is consistent with the well-known right hemispheric dominance for spatial functions (Serrien, Ivry, \& Swinnen, 2006; Ellison et al., 2004; Sainburg, 2002). Our TMS study also provides evidence for a specific role of right STG in monitoring this type of spatio-temporal coordination because virtual lesions of this area hampered the subjects' ability to cope with speed changes in the coordination task but not the independent one.

Our fMRI results also suggest that right SMA plays a specific role in bimanual coordination. This area has long been regarded as a critical region for controlling bimanual movements (Debaere, Wenderoth, Sunaert, Van Hecke, \& Swinnen, 2004a; Steyvers et al., 2003; Donchin et al., 2002; Meyer-Lindenberg et al., 2002; Obhi, Haggard, Taylor, \& Pascual-Leone, 2002; Serrien, Strens, Oliviero, \& Brown, 2002; Immisch, Waldvogel, van Gelderen, \& Hallett, 2001; Stephan, Binkofski, Halsband, et al., 1999; Stephan, Binkofski, Posse, Seitz, \& Freund, 1999; Sadato et al., 1997; Brinkman, 1981, 1984; Laplane, Talairach, Meininger, Bancaud, \& Orgogozo, 1977). The extensive connections between SMA and M1 on both sides and between both SMA (Liu, Morel, Wannier, \& Rouiller, 2002; Rouiller et al., 1994; Porter \& Lemon, 1993) are consistent with this view. However, despite the fact that SMA is a plausible candidate for the implementation of the spatio-temporal goal into the appropriate bimanual motor commands, TMS-induced virtual lesions of this area failed to affect our bimanual tasks, a result that contrasts with previous rTMS studies (Steyvers et al., 2003; Meyer-Lindenberg et al., 2002; Obhi et al., 2002; Serrien et al., 2002). However, in these studies, rTMS specifically altered the most "complex" coordination pattern, making it difficult to distinguish between a role of SMA 
Figure 5. Mean beta weight and time course of signal change (\% baseline) obtained from regions participating in the coordination task as evidenced by the conjunction analysis: $[(\mathrm{C}-\mathrm{R}) \cap(\mathrm{C}-\mathrm{I})]$. Threshold set at $p<.001$ (uncorrected), except for the SMA in which the threshold was $p<.002$ (uncorrected) to get a sufficiently large cluster size ( $>50$ voxels). $\mathrm{C}=$ coordination; $\mathrm{I}=$ independence; $\mathrm{R}=$ rest.

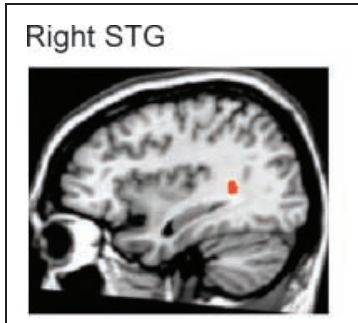

$36,-45,10$

Right M1

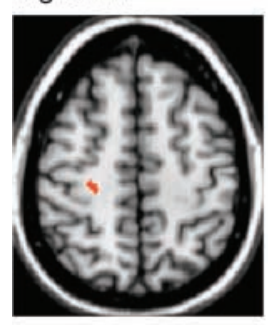

$31,-27,50$

Right SMA

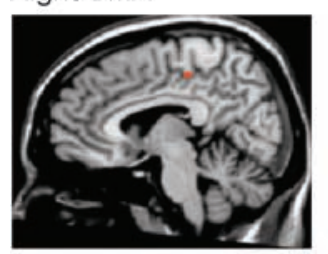

$4,-25,48$
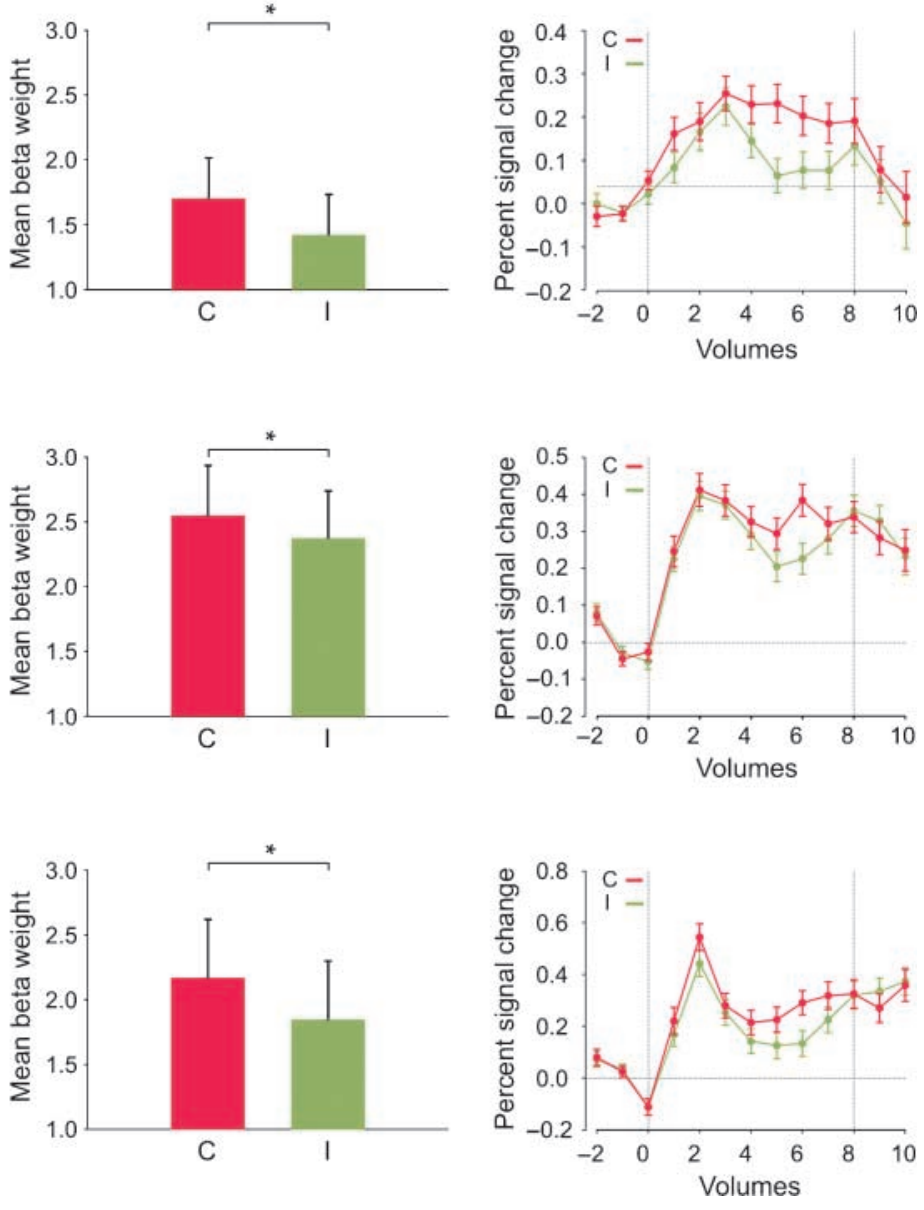

in controlling complex movements and a specific role in monitoring coordination from these studies. Alternatively, it is worth mentioning that, in the present study, at the beginning of each trial, subjects were given an explicit imperative cue regarding the required ratio to maintain in the coordination task; this was done to ensure similar performance levels despite different cognitive strategies in the two tasks. However, this approach, which decreased the need to retrieve the appropriate coupling ratio in the coordination task, might also have diminished the contribution of some areas, such as the SMA, to the coordination task. In addition, it is possible that because the SMA area is deeply buried in the interhemispheric sulcus, it is harder to interfere with its activity by using a reasonably low TMS intensity (Meyer-Lindenberg et al., 2002) or with the coil orientation we used. Finally, because other investigators have already managed to stimulate this region at lower intensities (Matsunaga et al., 2005; Civardi, Cantello, Asselman, \& Rothwell, 2001), it cannot be excluded that the movement parameters we measured were not sensitive enough to detect the effects of small SMA virtual lesions. Further investigations would, therefore, be necessary to isolate the specific contribution of SMA to bimanual coordination.

Finally, our fMRI results indicate that a third area, right $\mathrm{M} 1$, is more active in the coordination than in the indepen- dent task. However, surprisingly, we found that a virtual lesion of right M1 did not affect the coordination task but rather improved the independent task performance by enhancing the capacity of the left contralateral hand to cope with the speed changes. One possible explanation for this apparent contradiction is as follows: In the independent task, it is plausible that strong inhibitory interactions took place between the two motor cortices to minimize bimanual cross talk (Daffertschofer, Peper, \& Beek, 2005; Duque, Mazzocchio, et al., 2005; Ferbert et al., 1992). Consistently, interhemispheric interactions have been shown to vary with the features of the task at hand (Perez \& Cohen, 2008; Daffertschofer et al., 2005; Andres et al., 1999), and inhibition is known to be stronger from the dominant (left) to nondominant (right) hemisphere than in the opposite direction (Baumer et al., 2007; Duque et al., 2007; Netz, 1999). In the coordination task, this inhibition is likely to be released to allow a tight coordination between both hands; therefore, it is sensible to assume that the stronger activation we found in right M1 when contrasting the activation maps gathered in both conditions was due to a disinhibition in the coordinated task rather than an increased activation. This could explain the apparent contradiction between fMRI and TMS results. Indeed, the better performance found in the independent task following right 
M1 virtual lesions could have been caused by a change in the balance between inhibitory and facilitatory interhemispheric interactions occurring between both M1 (Duque et al., 2007, 2008; Duque, Hummel, et al., 2005; Ferbert et al., 1992), favoring the independence between the two hands. However, because this explanation remains largely speculative, assessing interhemispheric inhibitory interactions using a TMS paired-pulse design in the coordination and the independent tasks would be a very interesting issue for future investigation (Duque et al., 2007; Duque, Hummel, et al., 2005; Murase, Duque, Mazzocchio, \& Cohen, 2004). Hence, in contrast to the more straightforward interpretation of our fMRI results, the TMS results rather suggest that right M1 is not causally involved in the coordination aspect of bimanual hand movements, a conclusion that strengthens even further the benefit of combining fMRI and TMS.

It is noteworthy that, in the present study, all areas found more active in the coordination task were located in the nondominant hemisphere. This finding questions the long-held belief that the left dominant hemisphere is important for bimanual tasks and raises the possibility that the motor network underlying bimanual tasks depends on the nature of coordination in play. Here, we used a co- ordination task where subjects had to reach for a spatiotemporal bimanual goal. Consistently, the nondominant hemisphere is known to be "dominant" in dealing with such spatial information. In contrast, it is likely that such a hemispheric asymmetry would reverse in bimanual coordination tasks that do not rely as much on spatio-temporal cues.

As a final note, we would like to mention that even if our two tasks were designed in such a way that the feedback and the visual stimuli were identical in the two conditions, we cannot ascertain that subjects actually used this available information similarly in the two tasks. In particular, it is plausible that subjects generated their movements more internally in the coordination than in the independent task and that this slight difference might have also influenced our fMRI results. However, note that the brain regions evidenced by Debaere et al. (2003), as specifically involved in internally generated bimanual movements, differ from those we found in the coordination task, confirming that this factor cannot explain our fMRI and TMS results. In particular, Debaere et al. did not find any difference between STG or M1 activation in internally and externally generated bimanual movements. As far as SMA is concerned, we cannot rule out the possibility that its stronger activation in the coordination condition
Figure 6. Mean beta weight and time course of signal change (\% baseline) obtained from regions participating in the independent task as evidenced by the conjunction analysis: $[(\mathrm{I}-\mathrm{R}) \cap(\mathrm{I}-\mathrm{C})]$. Threshold set at $p<.001$ (uncorrected). $\mathrm{C}=$ coordination; $\mathrm{I}=$ independence; $\mathrm{R}=$ rest; MTG $=$ middle temporal gyrus; IPG $=$ inferior parietal gyrus; $F G$ = fusiform gyrus.

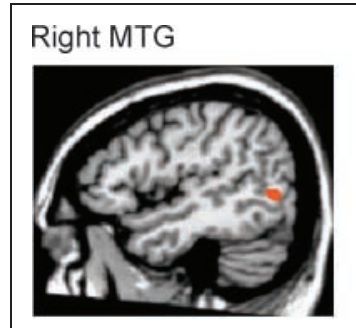

$48,-65,2$

Right IPG

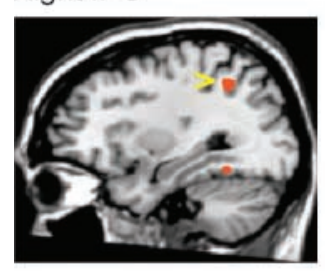

$30,-51,41$

Right FG

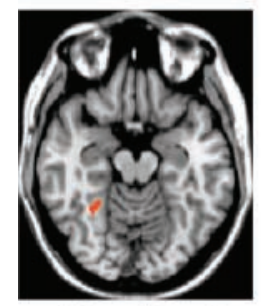

$29,-48,-13$
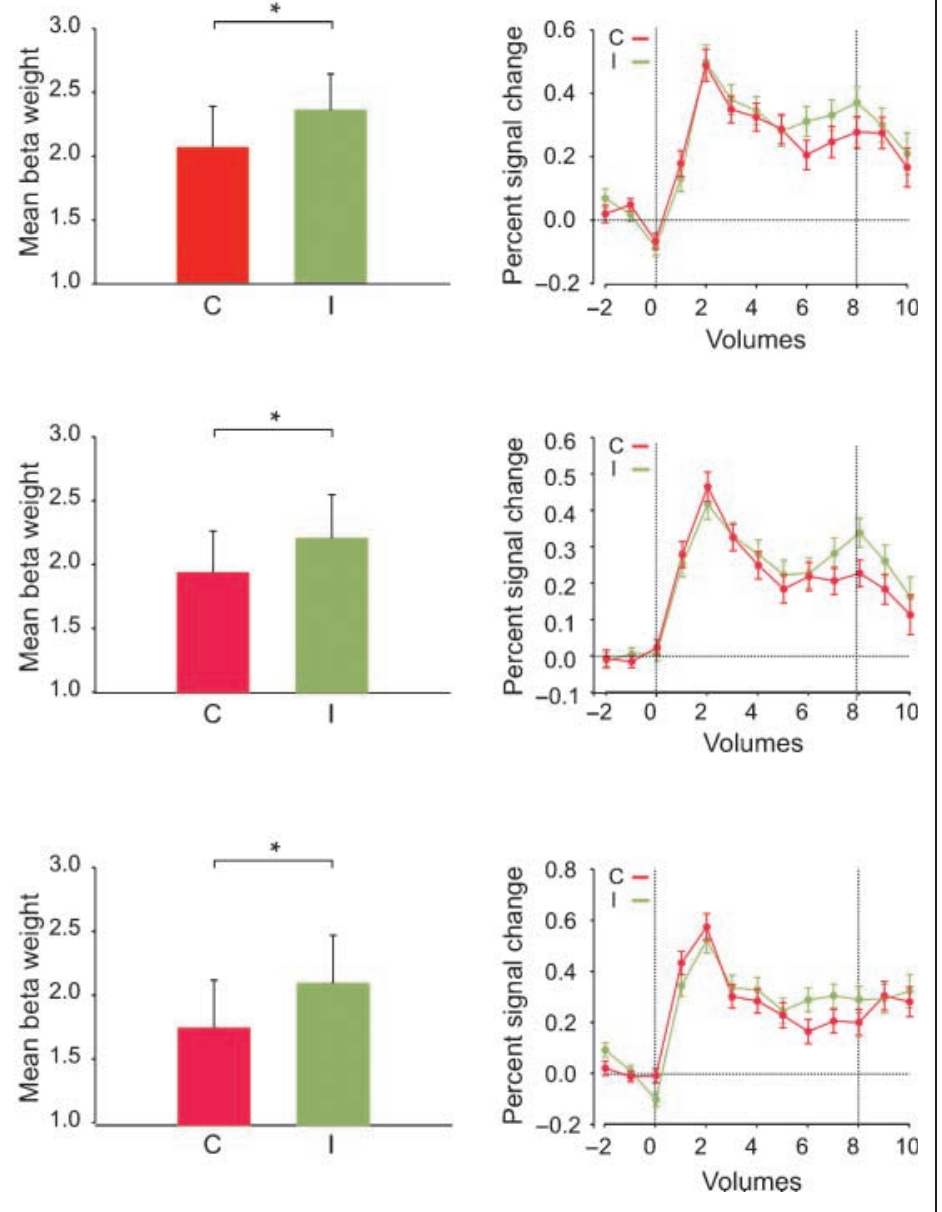


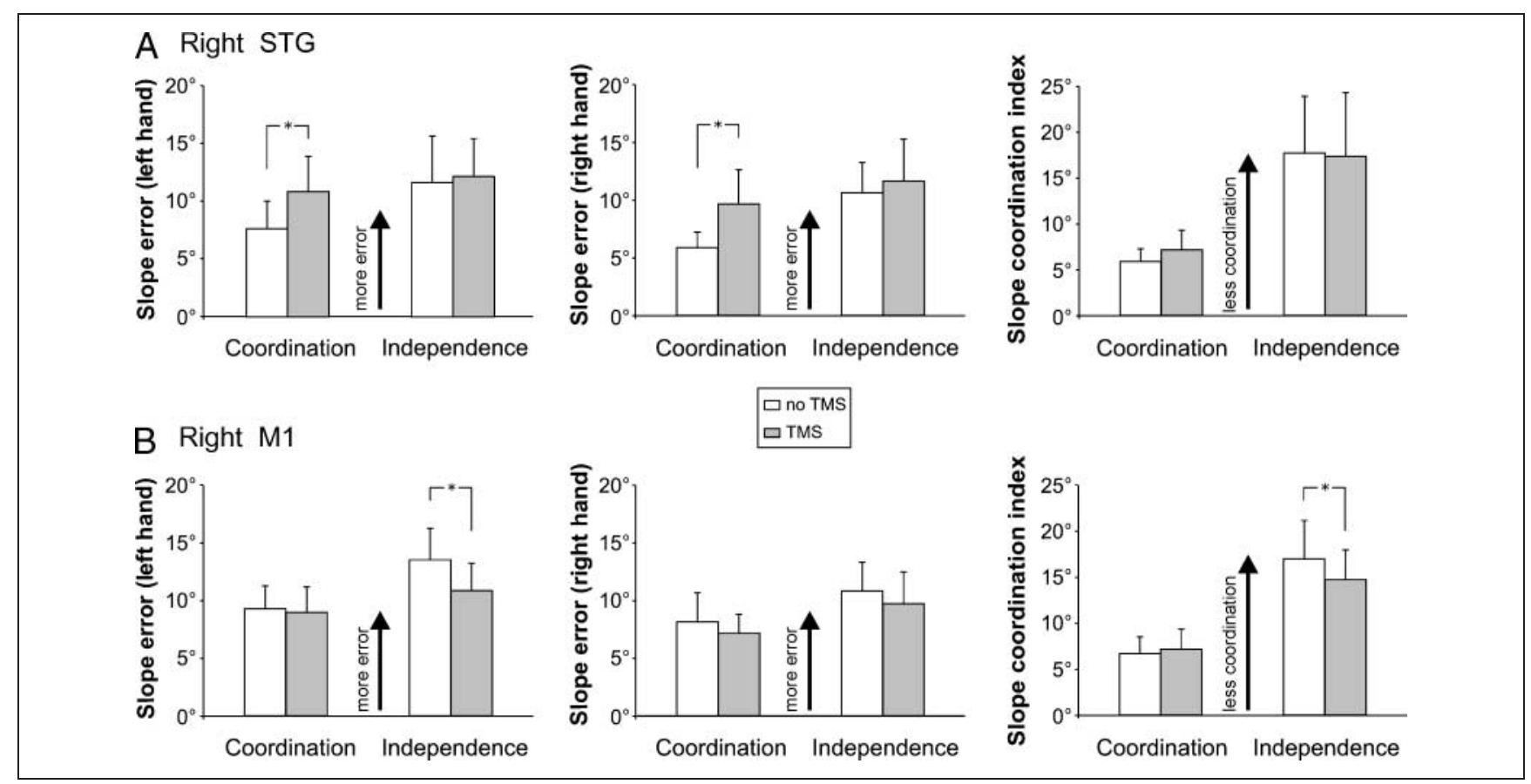

Figure 7. Mean and $S D$ of the left and the right hand hand slope error (left and middle traces, respectively) and of the slope coordination index (right trace) in the two bimanual tasks (coordination and independence), with (gray bars) and without TMS (white bars) over the right STG (A) and the right M1 (B).

was partly due to a higher reliance on internally generate movements in this task (Debaere et al., 2003; Cunnington, Windischberger, Deecke, \& Moser, 2002; Deiber, Honda, Ibanez, Sadato, \& Hallett, 1999).

\section{Methodological Consideration: The Bimanual Tasks}

A key point of the present study was to isolate the control mechanisms specifically involved in the "coordination aspect" of bimanual movements; the coordination aspect refers to the processes underlying interactions that occurred between both hands to achieve the bimanual goal, that is, a given speed ratio between hands. Although this aspect is one of the most important prerequisites to perform efficient bimanual movements, it is also the trickiest one to investigate experimentally because of the difficulty to isolate it from all other variables known to affect individual movements during bimanual actions (complexity, learning stage, feedback procedure, etc.). Here, we attempted to solve this problem by using an original experimental design in which we used two bimanual tasks differing only from the nature of the cognitive control underlying their performance: In the coordination task, subjects were explicitly asked to reach a unique bimanual goal, namely, to maintain a given speed ratio between the two hand motions, whereas in the independent task, the two hands had to remain as uncoupled as possible to reach distinct goals, that is, different speeds. Importantly, we minimized the differences between the two tasks for all other aspects: hand speed, performance level, visual display, feedback procedure, etc.

Our main concern in designing these tasks was to reduce the possibility that subjects could also use, even if not explicitly provided, the hand speed ratios to perform the independent task, hampering the comparison between the two conditions. To avoid this, we selected, for the independent task, hand speeds that led to noninteger ratios, varying from 1.1 to 4.8; in contrast to the ratios used in the coordination task, these noninteger ratios are extremely difficult to produce explicitly, even for expert musicians (Ullen et al., 2003; Peper, Beek, \& van Wieringen, 1995; Summers, Todd, \& Kim, 1993). In addition, the hand speed ratio was modified twice during each trial (once in the TMS experiment) to make it even more difficult for subjects to use a coordination strategy based on the hand speed ratio in the independent condition. Therefore, it is sensible to assume that subjects had no other option than using distinct strategies to perform the independent and the coordinated tasks. This assumption was supported by the subtle differences we found between the performances of the two tasks. Indeed, despite an identical amount of hand speed error in both tasks, the coordination index was larger in the independent than in the coordination task, an observation consistent with the hypothesis that subjects did not control this variable explicitly in the independent task as they did in the coordination task. Additionally, in the coordination task, the hand speed error was always smaller for the fastest hand-likely the leading one (Johansson et al., 2006)—when compared with the other hand. Such an interaction between the speed errors 
in the two hands was not found in the independent task, suggesting further that, in this condition, the two hand movements were controlled separately. Altogether, these observations corroborate the view that subjects used different cognitive strategies to perform the two tasks, yet identical in most other respects.

Moreover, it is noteworthy that the fact that subjects were able to perform the independent task with the same level of accuracy as the coordination task confirms that interference during independent tasks can be attenuated by training, possibly by strengthening strategies that permit to cancel out the bimanual cross talk (Oliveira \& Ivry, 2008; Rosenbaum et al., 2006; Diedrichsen et al., 2001).

Finally, in the present study, we only focused on one particular type of bimanual coordination, based on the explicit control of an integrated spatio-temporal goal and, therefore, our results are unlikely to generalize to other coordination strategies such as those, for example, in which bimanual movements are synchronized by external visual goals. In addition, we cannot completely rule out the possibility that subjects somewhat relied on such visual coordination strategy to perform the independent task, although we minimized this option by using a distinct feedback for each hand, and the behavioral results also support the use of an uncoupled strategy in the independent task. In any case, we think our experimental design was optimal to highlight the neural correlates of a specific coordination strategy based on the integration of a spatio-temporal goal.

\section{Conclusions}

Bimanual coordination is critical for most daily motor actions, and still, its neural correlates remain unclear. A better knowledge of how hand representations interact to achieve complex tasks could yield substantial improvement in the fields of rehabilitation, sport science, cortical prosthetics, and possibly neurosurgery.

The combined use of fMRI and TMS enabled us to demonstrate the specific contribution of some cortical areas in the control of the coordination aspect of bimanual movements. We propose that right STG plays a role in monitoring bimanual spatio-temporal goals. Regarding right SMA, its contribution to the implementation of these spatio-temporal goals into adequate motor commands is plausible but would require further investigation. Finally, in contrast to the fMRI results, the TMS study suggests that right M1 is not causally involved in the coordination aspect of bimanual movements but that its activity is modulated by inhibitory influences, likely to be stronger in the independent than in the coordination task.

\section{Acknowledgments}

We are grateful to M. Penta, C. Schiltz, and N. Wenderoth for helping us with the experimental design and A. Heinecke with the analysis of the imaging data. We would also like to thank
R. Ivry and J. Diedrichsen for their critical comments on an earlier version of this manuscript. This work was supported by grants from the ARC grant 07/12-007 (Communauté Française de Belgique-Actions de Recherche Concertées), the "Fonds Spéciaux de Recherche" (FSR) of the Université catholique de Louvain, the "Fonds de la Recherche Scientifique Médicale" (FRSM), and the "Fondation Médicale Reine Elisabeth" (FMRE). J. D. and M. D. are research fellows at the Belgian National Funds for Scientific Research (FNRS).

Reprint requests should be sent to Julie Duque, Laboratory of Neurophysiology, Institute of Neuroscience, Université catholique de Louvain, Avenue Hippocrate, 54, 1200 Brussels, Belgium, or via e-mail: julie.duque@uclouvain.be.

\section{REFERENCES}

Aboitiz, F., Ide, A., \& Olivares, R. (2003). Corpus callosum morphology in relation to cerebral asymmetries in the postmortem human. In The parallel brain: The cognitive neuroscience of the corpus callosum (pp. 33-46). Cambridge, MA: MIT Press.

Andres, F. G., Mima, T., Schulman, A. E., Dichgans, J., Hallett, M., \& Gerloff, C. (1999). Functional coupling of human cortical sensorimotor areas during bimanual skill acquisition. Brain, 122, 855-870.

Baumer, T., Dammann, E., Bock, F., Kloppel, S., Siebner, H. R., \& Munchau, A. (2007). Laterality of interhemispheric inhibition depends on handedness. Experimental Brain Research, 180, 195-203.

Boynton, G. M., Engel, S. A., Glover, G. H., \& Heeger, D. J. (1996). Linear systems analysis of functional magnetic resonance imaging in human V1. Journal of Neuroscience, 16, 4207-4221.

Brinkman, C. (1981). Lesions in supplementary motor area interfere with a monkey's performance of a bimanual coordination task. Neuroscience Letters, 27, 267-270.

Brinkman, C. (1984). Supplementary motor area of the monkey's cerebral cortex: Short- and long-term deficits after unilateral ablation and the effects of subsequent callosal section. Journal of Neuroscience, 4, 918-929.

Civardi, C., Cantello, R., Asselman, P., \& Rothwell, J. C. (2001). Transcranial magnetic stimulation can be used to test connections to primary motor areas from frontal and medial cortex in humans. Neuroimage, 14, 1444-1453.

Cunnington, R., Windischberger, C., Deecke, L., \& Moser, E. (2002). The preparation and execution of self-initiated and externally-triggered movement: A study of event-related fMRI. Neuroimage, 15, 373-385.

Daffertschofer, A., Peper, C. L., \& Beek, P. J. (2005). Stabilization of bimanual coordination due to active interhemispheric inhibition: A dynamical account. Biological Cybernetics, 92, 101-109.

Davare, M., Andres, M., Clerget, E., Thonnard, J. L., \& Olivier, E. (2007). Temporal dissociation between hand shaping and grip force scaling in the anterior intraparietal area. Journal of Neuroscience, 27, 3974-3980.

Davare, M., Andres, M., Cosnard, G., Thonnard, J. L., \& Olivier, E. (2006). Dissociating the role of ventral and dorsal premotor cortex in precision grasping. Journal of Neuroscience, 26, 2260-2268.

De Weerd, P., Reinke, K., Ryan, L., McIsaac, T., Perschler, P., Schnyer, D., et al. (2003). Cortical mechanisms for acquisition and performance of bimanual motor sequences. Neuroimage, 19, 1405-1416.

Debaere, F., Swinnen, S. P., Beatse, E., Sunaert, S., Van Hecke, P., \& Duysens, J. (2001). Brain areas involved in interlimb coordination: A distributed network. Neuroimage, 14, 947-958. 
Debaere, F., Wenderoth, N., Sunaert, S., Van Hecke, P., \& Swinnen, S. P. (2003). Internal vs external generation of movements: Differential neural pathways involved in bimanual coordination performed in the presence or absence of augmented visual feedback. Neuroimage, 19, 764-776.

Debaere, F., Wenderoth, N., Sunaert, S., Van Hecke, P., \& Swinnen, S. P. (2004a). Cerebellar and premotor function in bimanual coordination: Parametric neural responses to spatiotemporal complexity and cycling frequency. Neuroimage, 21, 1416-1427.

Debaere, F., Wenderoth, N., Sunaert, S., Van Hecke, P., \& Swinnen, S. P. (2004b). Changes in brain activation during the acquisition of a new bimanual coodination task. Neuropsychologia, 42, 855-867.

Deiber, M. P., Honda, M., Ibanez, V., Sadato, N., \& Hallett, M. (1999). Mesial motor areas in self-initiated versus externally triggered movements examined with fMRI: Effect of movement type and rate. Journal of Neurophysiology, 81, 3065-3077.

Diedrichsen, J., Hazeltine, E., Kennerley, S., \& Ivry, R. B. (2001). Moving to directly cued locations abolishes spatial interference during bimanual actions. Psychological Science, 12, 493-498.

Donchin, O., Gribova, A., Steinberg, O., Mitz, A. R., Bergman, H., \& Vaadia, E. (2002). Single-unit activity related to bimanual arm movements in the primary and supplementary motor cortices. Journal of Neurophysiology, 88, 3498-3517.

Duque, J., Hummel, F., Celnik, P., Murase, N., Mazzocchio, R., \& Cohen, L. G. (2005). Transcallosal inhibition in chronic subcortical stroke. Neuroimage, 28, 940-946.

Duque, J., Mazzocchio, R., Dambrosia, J., Murase, N., Olivier, E., \& Cohen, L. G. (2005). Kinematically specific interhemispheric inhibition operating in the process of generation of a voluntary movement. Cerebral Cortex, $15,588-593$.

Duque, J., Mazzocchio, R., Stefan, K., Hummel, F., Olivier, E., \& Cohen, L. G. (2008). Memory formation in the motor cortex ipsilateral to a training hand. Cerebral Cortex, $18,1395-1406$.

Duque, J., Murase, N., Celnik, P., Hummel, F., Harris-Love, M., Mazzocchio, R., et al. (2007). Intermanual differences in movement-related interhemispheric inhibition. Journal of Cognitive Neuroscience, 19, 204-213.

Ellison, A., Schindler, I., Pattison, L. L., \& Milner, A. D. (2004). An exploration of the role of the superior temporal gyrus in visual search and spatial perception using TMS. Brain, 127, 2307-2315.

Ferbert, A., Priori, A., Rothwell, J. C., Day, B. L., Colebatch, J. G., \& Marsden, C. D. (1992). Interhemispheric inhibition of the human motor cortex. Journal of Physiology, 453, 525-546.

Franz, E. A. (1997). Spatial coupling in the coordination of complex actions. Quarterly Journal of Experimental Psychology A, 50, 684-704.

Franz, E. A., Zelaznik, H. N., Swinnen, S. S., \& Walter, C. (2001). Spatial conceptual influences on the coordination of bimanual actions: When a dual task becomes a single task. Journal of Motor Behavior, 33, 103-112.

Friston, K. J., Frith, C. D., Turner, R., \& Frackowiak, R. S. (1995). Characterizing evoked hemodynamics with fMRI. Neuroimage, 2, 157-165.

Friston, K. J., Penny, W. D., \& Glaser, D. E. (2005). Conjunction revisited. Neuroimage, 25, 661-667.

Genovese, C. R., Lazar, N. A., \& Nichols, T. (2002). Thresholding of statistical maps in functional neuroimaging using the false discovery rate. Neuroimage, 15, 870-878.
Gharabaghi, A., Fruhmann Berger, M., Tatagiba, M., \& Karnath, H. O. (2006). The role of the right superior temporal gyrus in visual search-insights from intraoperative electrical stimulation. Neuropsychologia, 44, 2578-2581.

Immisch, I., Waldvogel, D., van Gelderen, P., \& Hallett, M. (2001). The role of the medial wall and its anatomical variations for bimanual antiphase and in-phase movements. Neuroimage, 14, 674-684.

Jantzen, K. J., Oullier, O., \& Scott Kelso, J. A. (2008). Neuroimaging coordination dynamics in the sport sciences. Methods, 45, 325-335.

Johansson, R. S., Theorin, A., Westling, G., Andersson, M., Ohki, Y., \& Nyberg, L. (2006). How a lateralized brain supports symmetrical bimanual tasks. PLoS Biology, 4, e158.

Karnath, H. O. (2001). New insights into the functions of the superior temporal cortex. Nature Reviews Neuroscience, 2, 568-576.

Karnath, H. O., Ferber, S., \& Himmelbach, M. (2001). Spatial awareness is a function of the temporal not the posterior parietal lobe. Nature, 411, 950-953.

Karnath, H. O., Zopf, R., Johannsen, L., Berger, M. F., Nagele, T., \& Klose, U. (2005). Normalized perfusion MRI to identify common areas of dysfunction: Patients with basal ganglia neglect. Brain, 128, 2462-2469.

Laplane, D., Talairach, J., Meininger, V., Bancaud, J., \& Orgogozo, J. M. (1977). Clinical consequences of corticectomies involving the supplementary motor area in man. Journal of the Neurological Sciences, 34, 301-314.

Liu, J., Morel, A., Wannier, T., \& Rouiller, E. M. (2002). Origins of callosal projections to the supplementary motor area (SMA): A direct comparison between pre-SMA and SMA-proper in macaque monkeys. Journal of Comparative Neurology, 443, 71-85.

Matsunaga, K., Maruyama, A., Fujiwara, T., Nakanishi, R., Tsuji, S., \& Rothwell, J. C. (2005). Increased corticospinal excitability after $5 \mathrm{~Hz}$ rTMS over the human supplementary motor area. Journal of Physiology, 562, 295-306.

Mayville, J. M., Jantzen, K. J., Fuchs, A., Steinberg, F. L., \& Kelso, J. A. (2002). Cortical and subcortical networks underlying syncopated and synchronized coordination revealed using fMRI. Functional magnetic resonance imaging. Human Brain Mapping, 17, 214-229.

Mechsner, F., Kerzel, D., Knoblich, G., \& Prinz, W. (2001). Perceptual basis of bimanual coordination. Nature, 414, 69-73.

Meister, I. G., Wienemann, M., Buelte, D., Grunewald, C., Sparing, R., Dambeck, N., et al. (2006). Hemiextinction induced by transcranial magnetic stimulation over the right temporo-parietal junction. Neuroscience, 142, 119-123.

Meyer-Lindenberg, A., Ziemann, U., Hajak, G., Cohen, L., \& Berman, K. F. (2002). Transitions between dynamical states of differing stability in the human brain. Proceedings of the National Academy of Sciences, U.S.A., 99, 10948-10953.

Mort, D. J., Malhotra, P., Mannan, S. K., Rorden, C., Pambakian, A., Kennard, C., et al. (2003). The anatomy of visual neglect. Brain, 126, 1986-1997.

Murase, N., Duque, J., Mazzocchio, R., \& Cohen, L. G. (2004). Influence of interhemispheric interactions on motor function in chronic stroke. Annals of Neurology, 55, 400-409.

Netz, J. (1999). Asymmetry in transcallosal inhibition. Electroencephalography and Clinical Neurophysiology. Supplement, 51, 137-144.

Nichols, T., Brett, M., Andersson, J., Wager, T., \& Poline, J. B. (2005). Valid conjunction inference with the minimum statistic. Neuroimage, 25, 653-660. 
Noirhomme, Q., Ferrant, M., Vandermeeren, Y., Olivier, E., Macq, B., \& Cuisenaire, O. (2004). Registration and real-time visualization of transcranial magnetic stimulation with 3-D MR images. IEEE Transactions on Biomedical Engineering, 51, 1994-2005.

Obhi, S. S., Haggard, P., Taylor, J., \& Pascual-Leone, A. (2002). rTMS to the supplementary motor area disrupts bimanual coordination. Motor Control, 6, 319-332.

Oliveira, F. T. P., \& Ivry, R. B. (2008). The representation of action: Insights from bimanual coordination. Current Directions in Psychological Science, 17, 130-135.

Oullier, O., Jantzen, K. J., Steinberg, F. L., \& Kelso, J. A. (2005). Neural substrates of real and imagined sensorimotor coordination. Cerebral Cortex, 15, 975-985.

Peper, C. E., Beek, P. J., \& van Wieringen, P. C. (1995). Frequency-induced phase transitions in bimanual tapping. Biological Cybernetics, 73, 301-309.

Perez, M. A., \& Cohen, L. G. (2008). Mechanisms underlying functional changes in the primary motor cortex ipsilateral to an active hand. Journal of Neuroscience, 28, 5631-5640.

Porter, R., \& Lemon, R. N. (1993). Corticospinal function and voluntary movement. Monographs of the Physiological Society, 45.

Price, C. J., \& Friston, K. J. (1997). Cognitive conjunction: A new approach to brain activation experiments. Neuroimage, 5, 261-270.

Puttemans, V., Wenderoth, N., \& Swinnen, S. P. (2005). Changes in brain activation during the acquisition of a multifrequency bimanual coordination task: From the cognitive stage to advanced levels of automaticity. Journal of Neuroscience, 25, 4270-4278.

Rosenbaum, D. A., Dawson, A. M., \& Challis, J. H. (2006). Haptic tracking permits bimanual independence. Journal of Experimental Psychology: Human Perception and Performance, 32, 1266-1275.

Rossini, P. M., Barker, A. T., Berardelli, A., Caramia, M. D., Caruso, G., Cracco, R. Q., et al. (1994). Non-invasive electrical and magnetic stimulation of the brain, spinal cord and roots: Basic principles and procedures for routine clinical application. Report of an IFCN committee. Electroencephalography and Clinical Neurophysiology, 91, 79-92.

Rouiller, E. M., Babalian, A., Kazennikov, O., Moret, V., Yu, X. H., \& Wiesendanger, M. (1994). Transcallosal connections of the distal forelimb representations of the primary and supplementary motor cortical areas in macaque monkeys. Experimental Brain Research, 102, 227-243.

Sadato, N., Yonekura, Y., Waki, A., Yamada, H., \& Ishii, Y. (1997). Role of the supplementary motor area and the right premotor cortex in the coordination of bimanual finger movements. Journal of Neuroscience, 17, 9667-9674.

Sainburg, R. L. (2002). Evidence for a dynamic-dominance hypothesis of handedness. Experimental Brain Research, 142, 241-258.

Serrien, D. J., Bogaerts, H., Suy, E., \& Swinnen, S. P. (1999). The identification of coordination constraints across planes of motion. Experimental Brain Research, 128, $250-255$

Serrien, D. J., Ivry, R. B., \& Swinnen, S. P. (2006). Dynamics of hemispheric specialization and integration in the context of motor control. Nature Reviews Neuroscience, 7, 160-166.

Serrien, D. J., Strens, L. H., Oliviero, A., \& Brown, P. (2002). Repetitive transcranial magnetic stimulation of the supplementary motor area (SMA) degrades bimanual movement control in humans. Neuroscience Letters, 328, 89-92.

Stephan, K. M., Binkofski, F., Halsband, U., Dohle, C., Wunderlich, G., Schnitzler, A., et al. (1999). The role of ventral medial wall motor areas in bimanual co-ordination. A combined lesion and activation study. Brain, 122, 351-368.

Stephan, K. M., Binkofski, F., Posse, S., Seitz, R. J., \& Freund, H. J. (1999). Cerebral midline structures in bimanual coordination. Experimental Brain Research, 128, $243-249$

Steyvers, M., Etoh, S., Sauner, D., Levin, O., Siebner, H. R., Swinnen, S. P., et al. (2003). High-frequency transcranial magnetic stimulation of the supplementary motor area reduces bimanual coupling during anti-phase but not in-phase movements. Experimental Brain Research, 151, 309-317.

Summers, J. J., Todd, J. A., \& Kim, Y. H. (1993). The influence of perceptual and motor factors on bimanual coordination in a polyrhythmic tapping task. Psychological Research, 55, 107-115.

Swinnen, S. P. (2002). Intermanual coordination: From behavioural principles to neural-network interactions. Nature Reviews Neuroscience, 3, 348-359.

Swinnen, S. P., \& Wenderoth, N. (2004). Two hands, one brain: Cognitive neuroscience of bimanual skill. Trends in Cognitive Sciences, 8, 18-25.

Talairach, J., \& Tournoux, P. (1988). Co-planar stereotaxic atlas of the human brain, 3-dimensional proportional system: An approach to cerebral imaging. New York: Thieme.

Thiebaut de Schotten, M., Urbanski, M., Duffau, H., Volle, E., Levy, R., Dubois, B., et al. (2005). Direct evidence for a parietal-frontal pathway subserving spatial awareness in humans. Science, 309, 2226-2228.

Toyokura, M., Muro, I., Komiya, T., \& Obara, M. (1999). Relation of bimanual coordination to activation in the sensorimotor cortex and supplementary motor area: Analysis using functional magnetic resonance imaging. Brain Research Bulletin, 48, 211-217.

Ullen, F., Forssberg, H., \& Ehrsson, H. H. (2003). Neural networks for the coordination of the hands in time. Journal of Neurophysiology, 89, 1126-1135.

Wassermann, E. M. (1998). Risk and safety of repetitive transcranial magnetic stimulation: Report and suggested guidelines from the International Workshop on the Safety of Repetitive Transcranial Magnetic Stimulation, June 5-7, 1996. Electroencephalography and Clinical Neurophysiology, 108, 1-16.

Wenderoth, N., Debaere, F., Sunaert, S., \& Swinnen, S. P. (2005a). The role of anterior cingulate cortex and precuneus in the coordination of motor behaviour. European Journal of Neuroscience, 22, 235-246.

Wenderoth, N., Debaere, F., Sunaert, S., \& Swinnen, S. P. (2005b). Spatial interference during bimanual coordination: Differential brain networks associated with control of movement amplitude and direction. Human Brain Mapping, 26, 286-300.

Wenderoth, N., Debaere, F., Sunaert, S., van Hecke, P., \& Swinnen, S. P. (2004). Parieto-premotor areas mediate directional interference during bimanual movements. Cerebral Cortex, 14, 1153-1163.

Wenderoth, N., Puttemans, V., Vangheluwe, S., \& Swinnen, S. P. (2003). Bimanual training reduces spatial interference. Journal of Motor Behavior, 35, 296-308. 